\title{
Knowledge and the Norm of Assertion: A Simple Test ${ }^{*}$
}

\author{
John Turri \\ john.turri@gmail.com
}

Abstract: An impressive case has been built for the hypothesis that knowledge is the norm of as sertion, otherwise known as the knowledge account of assertion. According to the knowledge account, you should assert something only if you know that it's true. A wealth of observational data supports the knowledge account, and some recent empirical results lend further, indirect support. But the knowledge account has not yet been tested directly. This paper fills that gap by reporting the results of such a test. The knowledge account passes with flying colors.

keywords: knowledge, assertion, norms, social cognition, belief, evidence, experimental philosophy

\section{Introduction}

All of us are intimately familiar with the practice of assertion. We've engaged in it for as long as we can remember, as have all the people in our lives. Others provide us with much of the infor-

\footnotetext{
* This is the penultimate version of a paper forthcoming in Synthese. Please cite the final, published version if possible.
} 
mation we rely on every day, and assertion is the main way we communicate information to one another. Given that we rely so pervasively on assertions, it's natural to wonder, what standard should assertions measure up to? What is the norm of assertion?

Social observation provides a wealth of clues about the norm of assertion. These observations suggest an important normative connection between what you know and what you should assert (for overviews see Benton, 2012; Turri, 2013a; for earlier relevant work, see Unger, 1975; Williamson, 2000). Consider just three of the most suggestive clues. First, knowledge seems to be implicated in prompting people to make assertions, abstaining from making assertions, and challenging assertions. We can prompt someone to make an assertion by asking them "What time is it?" or, equally well, by asking "Do you know what time it is?" Competent speakers respond to the two questions similarly. Second, we can abstain from answering a question by saying "Sorry, I don't know," even when the content of the question has nothing to do with you or what you know. And, third, we can challenge people’s assertions by saying "You don’t know that."

Social observation and introspection are natural places to start when investigating the norm of an important and familiar social practice like assertion. And these have gone a long way toward clarifying the norm of assertion. But some caution is warranted because introspection and social observation have well known limitations. Not infrequently we misunderstand the dynamics of social interaction and the source of our own actions and reactions (Milgram, 1974: 103-4; Nisbett \& Wilson, 1977; Schwitzgebel, 2008; Lieberman, 2013). Fortunately, the connection between knowledge and assertability also can be systemically investigated by established methods of experimental cognitive and social science. Controlled experimentation supplements intro- 
spection and social observation; it simultaneously builds on the insights they afford and overcomes their limitations, thereby increasing confidence that we have accurately identified the norm.

Two recent studies help shed some light on the issue. One study directly investigated whether the norm of assertion was, at the very least, "factive" or truth-entailing. A factive norm implies that only true assertions should be made (Turri, 2013b; see also Rakoczy \& Tomasello, 2009). This study was motivated by critics' repeated insistence that the norm of assertion cannot be factive, because factive norms are counterintuitive and mischaracterize the practice of assertion. Instead, critics propose, the norm must be belief, or good evidence, or belief supported by good evidence (e.g. Douven, 2006; Lackey, 2007; Hill \& Schechter, 2007; Kvanvig, 2009). But the results showed that the norm of assertion is overwhelmingly viewed as factive. The results also showed that critics' favorite sort of thought experiment, intended to pump intuitions against factive accounts, can cause serious performance errors when assessing norm-violation.

In another study that included over six hundred adult participants, people were asked to evaluate agents in many different situations, with different levels of evidence and with different amounts at stake (Turri \& Buckwalter, under review). In addition to answering whether the agent should inscribe some proposition, which implicates a written assertion, participants recorded judgments about many other things, including whether the proposition is true, whether the agent believes the proposition, whether the agent has good evidence for the proposition, and how important it is whether the proposition is true. Regression analyses showed that, of all these judgments, knowledge judgments best predicted judgments about whether the agent should inscribe 
the proposition.

Results from these two studies lend further support to the knowledge account. On the one hand, the most widespread criticism of the knowledge account is that it demands too much. In particular, many claim that the knowledge account mistakenly prohibits well-justified false assertions, which, we're told, are ordinarily viewed as perfectly acceptable. But this criticism is foun ded on a fiction, as demonstrated by the normative judgments of hundreds of ordinary speakers in carefully controlled experiments. On the other hand, the regression analyses suggest that knowledge judgments explain judgments of assertability, which is exactly what we would expect if ordinary speakers are implicitly aware that knowledge is the norm of assertion.

But neither experiment counts as a direct test of the knowledge account because neither in tervened on knowledge directly. That is, neither experiment manipulated the presence or absence of knowledge by including it as an independent variable in the experimental design (Scheines, 1997). This is important because if knowledge is the norm of assertion, then manipulating the presence or absence of knowledge should significantly affect people's assertability judgments. If this didn't happen, then it would be a serious problem for the knowledge account. By contrast, if manipulating knowledge does significantly affect assertability judgments, then it would be additional evidence for the knowledge account.

As already suggested, if knowledge is the norm of assertion, then full competence in the practice of assertion requires mastering the knowledge rule. Furthermore, competent speakers' judgments about assertability will be guided by their commitment to the knowledge rule. This does not imply that skilled practitioners have an explicit, articulable theory about what the rule 
is, or that they will answer "knowledge" if asked "what is the norm of assertion?" The assumption is not that skilled practitioners tend to be good theorists; rather, it's that patterns in their concrete, first-order intuitions and judgments manifest their skill in applying the relevant rules (Chomsky, 1957; Noveck \& Sperber, 2004).

\section{Experiment 1}

\section{Method}

Participants. One hundred ninety-three U.S. residents were tested (aged 18-72 years, mean age $=31.6$ years; $94 \%$ reporting English as a native language; 78 female). Participants were recruited and tested online (using Amazon Mechanical Turk and Qualtrics) and compensated $\$ 0.30$ for ap proximately 2 minutes of their time. Repeat participation was prevented. I excluded data from seven participants who failed comprehension questions, but including them does not affect the results reported below.

Materials and Procedure. Participants were randomly assigned to one of eight conditions in a 2 (Knows: knowledge/no-knowledge) $\times 4$ (vignette: coffee/avocado/married/taxes) betweensubjects design. Each participant read a single story about an agent, Mallory, who is asked a question. In the knowledge condition, Mallory "knows that" the relevant proposition is true. In the no-knowledge condition, Mallory "doesn't know whether" the relevant proposition is true. The difference across vignettes was not predicted to be significant and served merely as a robustness check. After the vignette, participants rated their agreement with the key test statement: 
Mallory "should say" that the relevant proposition is true. Responses were collected on a standard 6-point Likert scale, 1 ("strongly disagree") - 6 ("strongly agree"). After responding to the test statement, participants proceeded to a new screen and answered from memory a comprehension question about whether Mallory knew the relevant information. Participants filled out a brief demographic survey after testing. Questions were always asked in the same order and, except for the elements of Likert scales, options were rotated randomly. The appendix contains all the stimuli used in the experiment.

\section{Results}

If knowledge is the norm of assertion, then assignment to condition should have a large effect on whether participants judge that the agent should make the assertion. And this is exactly what happened. Preliminary analysis revealed that response to the test statement was not significantly affected by participant age, participant gender, or vignette. These factors entered into no main or interaction effects, so the analyses that follow collapse across them. An analysis of variance showed that whether Mallory knew significantly affected response to the test statement, $F(1$, $161)=515.79, \mathrm{p}<.001$, all tests two-tailed. A follow-up independent samples t-test showed that mean agreement was significantly higher in the knowledge condition $(\mathrm{M}=5.35, \mathrm{SD}=1.06)$ than in the no-knowledge condition $(\mathrm{M}=1.70, \mathrm{SD}=0.84), \mathrm{t}(191)=26.52, \mathrm{p}<.001, \mathrm{MD}=3.64,95 \%$ $\mathrm{CI}$ for $\mathrm{MD}=3.37$ to 3.91 . (See Fig. 1.) The magnitude of the mean difference was extremely large (Cohen's $d=3.84$ ). The mode response in the knowledge condition was "strongly agree" $(=6)$, and in the no-knowledge condition it was "strongly disagree" $(=1)$. 
In the knowledge condition, $94 \%$ of participants agreed to some degree with the test statement (i.e. selected "somewhat agree," "agree," or "strongly agree"), compared to $4 \%$ in the noknowledge condition. Binary logistic regression revealed that assignment to condition strongly predicted agreement with the test statement, explaining between $62.5 \%$ and $83.3 \%$ of variance and correctly predicting $95 \%$ of cases, $\chi^{2}(1, \mathrm{~N}=193)=189.12, \mathrm{p}<0.001$. By changing the agent's status from not knowing to knowing, the odds of agreeing that she should make the assertion increased by a factor of nearly 350 (or $34850 \%$ ), 95\% CI for odds ratio $=95$ to 1276 .
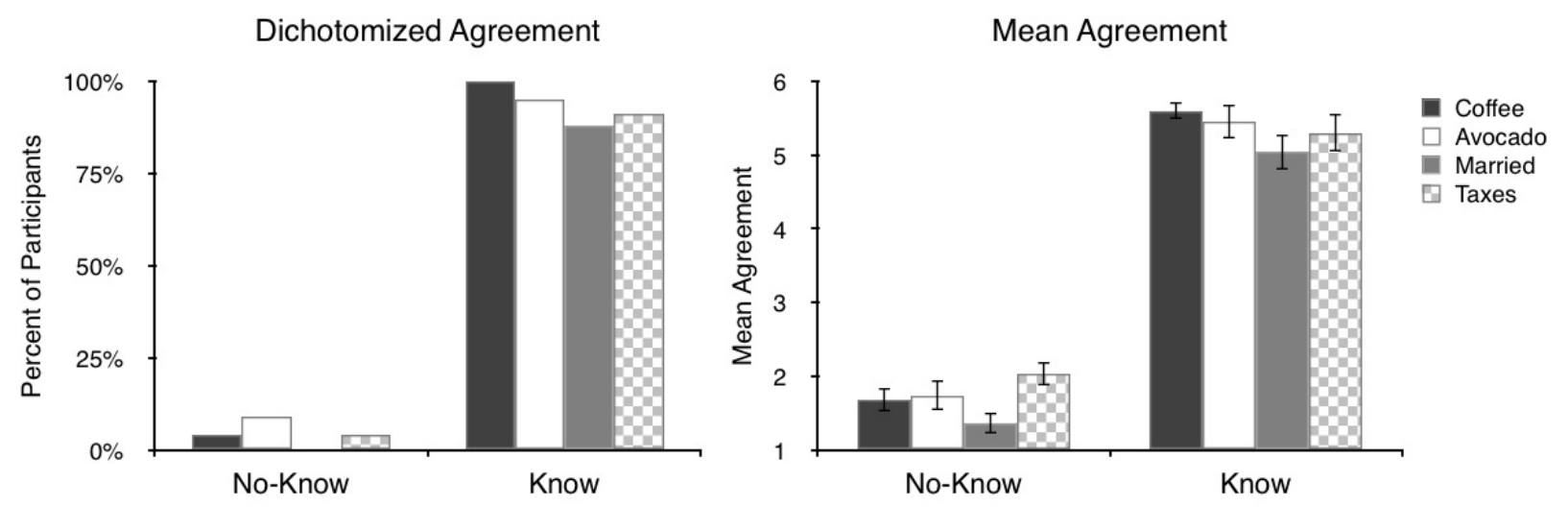

Fig 1. Experiment 1. Left panel: percent of participants agreeing to at least some degree ("somewhat agree," "agree," or "strongly agree") that the agent should make the assertion. Right panel: mean agreement with the test statement on a 1 (strongly disagree) - 6 (strongly agree) scale. Error bars represent $+/$ - one standard error of the mean.

\section{Discussion}

The results are exactly as the knowledge account would predict and, thus, strongly support the knowledge account. However, it might be objected that when participants are told that the agent 
“doesn't know" a proposition, they infer that the agent doesn't believe the proposition, or doesn't have evidence for the proposition. Because the stories above didn't explicitly state whether the agent believes or has evidence for the proposition, it cannot be definitively ruled out that partici pants in the no-knowledge conditions denied assertability because they inferred that belief or evidence was lacking, rather than knowledge. This interpretation is rendered unlikely by some of the research reviewed in the Introduction. Recall the study where, in addition to answering whether the agent should inscribe some proposition, participants also recorded judgments about whether the proposition was true, whether the agent believes the proposition, whether the agent has good evidence for the proposition, and whether the agent knows the proposition (Turri \& Buckwalter, under review). Regression analyses showed that knowledge judgments made the largest unique contribution to predicting people's judgments about whether the agent should inscribe the proposition. Thus it seems unlikely that, in the present experiment, participants in the no-knowledge conditions denied assertability because belief or evidence was lacking, rather than knowledge. Nevertheless, I conducted two additional experiments to directly address the objection.

\section{Experiments 2 and 3}

\section{Method}

Participants. There were ninety-five participants in Experiment 2 (aged 18-61 years, mean age $=32.1$ years; 96\% reporting English as a native language; 38 female) and eighty-five participants 
in Experiment 3 (aged 21-64 years, mean age $=32.9$ years; 96\% reporting English as a native language; 32 female). I excluded data from five participants who failed comprehension questions in Experiment 2 and fifteen participants in Experiment 3, but including them does not affect the results reported below. Participants were recruited and compensated in the same way as in Experiment 1.

Materials and Procedure. In each experiment, participants were randomly assigned to one of two conditions: knowledge and no-knowledge. Because there was no effect of vignette in Experiment 1, I used only a single vignette for these experiments (the coffee story from Experiment 1). There was one small change to the vignette in each case. This time, the final line of the story informed participants that Mallory "believes that" the relevant proposition is true in Experiment 2, whereas the final line informed them that Mallory "has evidence that" the proposition is true in Experiment 3. Thus, Mallory believes the proposition in each condition in Experiment 2, and she has evidence for the proposition in each condition for Experiment 3. In knowledge conditions, Mallory also knows that the proposition is true. In no-knowledge conditions, Mallory does not know that the proposition is true. After the vignette, participants rated their agreement with the key test statement — Mallory "should say" that the relevant proposition is true — using the same 6-point Likert scale as in Experiment 1. All other procedures were the same as in Experiment 1.

\section{Results}

In each experiment, preliminary analysis revealed that response to the test statement was not sig- 
nificantly affected by participant age or gender, so the analyses that follow collapse across these factors. In Experiment 2, an independent samples t-test showed that mean agreement was significantly higher in the knowledge condition $(\mathrm{M}=5.50, \mathrm{SD}=0.65)$ than in the no-knowledge condition $(\mathrm{M}=2.53, \mathrm{SD}=0.95), \mathrm{t}(81.2)=17.69, \mathrm{p}<.001, \mathrm{MD}=2.97,95 \% \mathrm{CI}$ for $\mathrm{MD}=2.64$ to 3.30 . (See Fig. 2.) The magnitude of the mean difference was extremely large (Cohen's $d=3.93)$. Similarly, in Experiment 3, an independent samples t-test showed that mean agreement was significantly higher in the knowledge condition $(\mathrm{M}=5.69, \mathrm{SD}=0.65)$ than in the no-knowledge condition $(\mathrm{M}=3.19, \mathrm{SD}=1.24), \mathrm{t}(49.23)=11.04, \mathrm{p}<.001, \mathrm{MD}=2.50,95 \% \mathrm{CI}$ for $\mathrm{MD}=2.09$ to 2.91. (See Fig. 2.) The magnitude of the mean difference was extremely large (Cohen's $d=3.15)$.
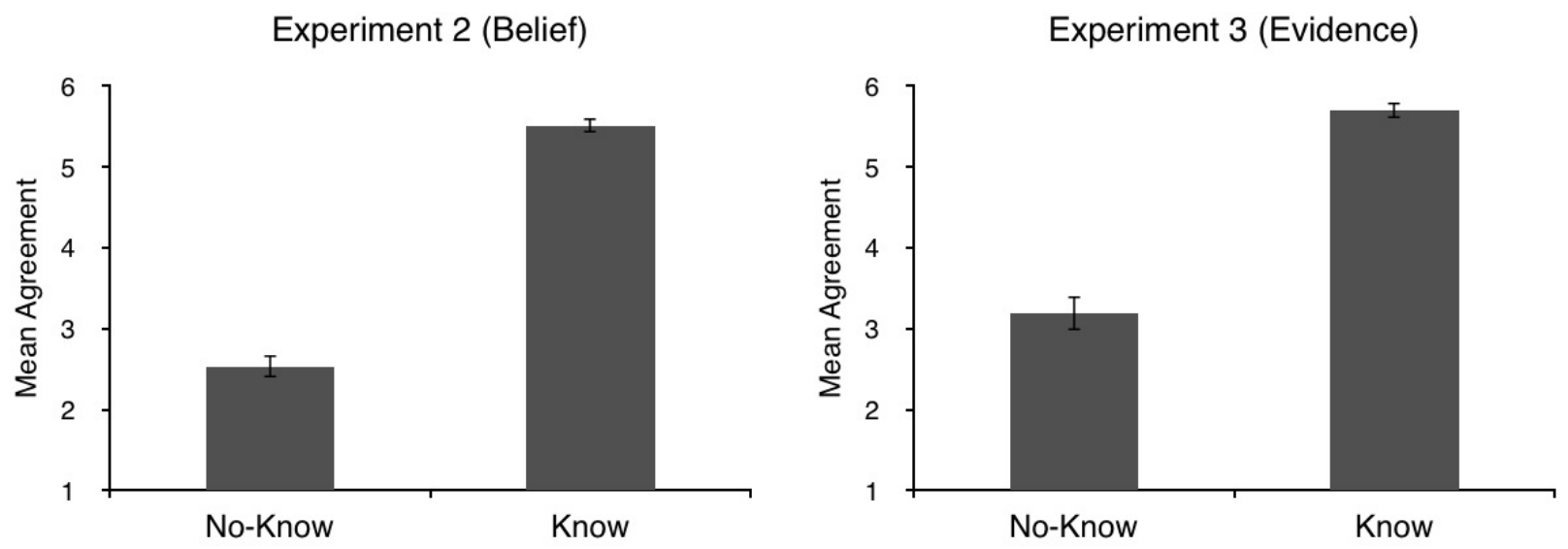

Fig 2. Experiments 2 and 3. Assertability ratings on a 1 (strongly disagree) - 6 (strongly agree) scale. Experiment 2 (left panel) held belief constant while varying the presence of knowledge. Experiment 3 (right panel) held possession of evidence constant while varying the presence of knowledge. Error bars represent $+/$ - one standard error of the mean. 


\section{Discussion}

The results again strongly support the knowledge account, replicating the main findings from Experiment 1 and addressing both concerns raised against it. In particular, the knowledge manipulation continued to have an extremely large effect on assertability judgments when the subject believed the relevant proposition and had evidence for it.

\section{Conclusion}

For decades researchers have steadily built a strong case that knowledge is the norm of assertion. According to this view, you should assert something only if you know that it's true. Prior evidence for the knowledge account included observational data and suggestive experimental results. But until now the knowledge account has not been directly tested. Ideally, the simplest and most direct test of a theory would be conducted first but, for whatever reason, it has been neglected in the burgeoning literature on the norms of assertion in general, and the knowledge account in particular.

In the spirit of the old adage, "Better late than never," this paper reports just such a test. The results were overwhelmingly favorable to the knowledge account. Intervening on whether an agent knows has an astonishingly large effect on judgments of assertability. Perhaps the most telling statistic is that by changing the agent's status from not knowing to knowing, the odds of judging that the agent should assert increased by a factor of nearly 350 (Experiment 1). For proponents of the knowledge account, this is definitely a result worth waiting for. 
In light of the abundant observational evidence, the indirect support from prior empirical findings, and the clear results from all three present studies, we should just go ahead and say it: knowledge is the norm of assertion.

The knowledge account reveals something deep and important about an absolutely central aspect of our lives as social beings, and it is one of the most significant contributions contemporary philosophy has made to our understanding of the human condition. Further progress in this area will begin not by asking whether knowledge is the norm of assertion, but instead by investigating, among other things, why knowledge plays this normative role. For instance, perhaps knowledge is the norm of assertion because the point of assertion is knowledge transmission, and an assertion can transmit knowledge only if it expresses knowledge (Turri, unpublished ms.). And although the present results suggest that neither gender nor age significantly affect judgments of assertability or its relationship to knowledge, further work could investigate whether there are interesting cross-cultural or developmental differences in judgments of assertability.

Acknowledgments — For helpful feedback, I thank Matt Benton, Wesley Buckwalter, David Rose, Angelo Turri, and two anonymous referees for Synthese. This research was supported by the Social Sciences and Humanities Research Council of Canada and an Early Researcher Award from the Ontario Ministry of Economic Development and Innovation.

word count: 2651 


\section{Appendix}

(Coffee) Mallory manages an independent coffee shop. One of her customers is interested in the history and culture of coffee. The customer asks Mallory whether the coffee is from Colombia. Mallory knows that/doesn't know whether the coffee is from Colombia. [Test statement: Mallory should say that the coffee is from Colombia.]

(Avocado) Mallory manages the local farmer's market. One of her employees is interested in improving the health of his diet. The employee asks Mallory whether avocados have vitamin

K. Mallory knows that/doesn't know whether avocados have vitamin K. [Test statement: Mallory should say that avocados have vitamin $\mathrm{K}$.]

(Married) Mallory is an intelligence analyst at the agency. One of her colleagues is interested in profiling an informant named Ivan. The colleague asks Mallory whether Ivan has ever been married. Mallory knows that/doesn't know whether Ivan has been married. [Test statement: Mallory should say that Ivan has been married.]

(Taxes) Mallory is a tax accountant at an insurance firm. One of her bosses is interested in decreasing the amount of taxes he pays. The boss asks Mallory whether he can deduct dental expenses. Mallory knows that/doesn’t know whether he can deduct dental expenses. [Test statement: Mallory should say that he can deduct dental expenses.]

(Comprehension question) Mallory ___ know the relevant information. (does / does not) 


\section{References}

Benton, M. A. (2012). Knowledge norms: assertion, belief, and action. Rutgers University, New Brunswick. PhD Thesis.

Chomsky, N. (1957). Syntactic structures. The Hague: Mouton.

Douven, I. (2006). Assertion, Knowledge, and Rational Credibility. Philosophical Review, 115(4), 449-485. doi:10.1215/00318108-2006-010

Hill, C., \& Schechter, J. (2007). Hawthorne's lottery puzzle and the nature of belief. Philosophi cal Issues, 17, 102-122.

Kvanvig, J. (2009). Assertion, knowledge, and lotteries. In D. Pritchard \& P. Greenough (Eds.), Williamson on knowledge (pp. 140-160). Oxford: Oxford University Press.

Lackey, J. (2007). Norms of assertion. Noûs, 41(4), 594-626.

Lieberman, M. D. (2013). Social: why our brains are wired to connect. New York: Crown Publishers.

Milgram, S. (1974). Obedience to authority: an experimental view. New York: Harper Perennial.

Noveck, I. A., \& Sperber, D. (2004). Experimental Pragmatics. Palgrave Macmillan.

Rakoczy, H., \& Tomasello, M. (2009). Done wrong or said wrong? Young children understand the normative directions of fit of different speech acts. Cognition, 113(2), 205-212. doi:10.1016/j.cognition.2009.07.013

Scheines, R. (1997). An introduction to causal inference. In V. McKim \& S. Turner (Eds.), Causality in crisis (pp. 185-2000). University of Notre Dame Press. 
Schwitzgebel, E. (2008). The Unreliability of Naive Introspection. Philosophical Review, 117(2), 245-273. doi:10.1215/00318108-2007-037

Turri, J. (2013a). Knowledge and suberogatory assertion. Philosophical Studies. doi:10.1007/ s11098-013-0112-z

Turri, J. (2013b). The test of truth: An experimental investigation of the norm of assertion. Cognition, 129(2), 279-291. doi:10.1016/j.cognition.2013.06.012

Turri, J. (Unpublished ms). Knowledge and the norm of assertion: an essay in philosophical science. University of Waterloo.

Turri, J. \& Buckwalter, W. (under review). Descartes's schism, Locke's reunion: completing the pragmatic turn in epistemology. University of Waterloo.

Unger, P. (1975). Ignorance: a case for skepticism. Oxford: Oxford University Press.

Williamson, T. (2000). Knowledge and its limits. Oxford: Oxford University Press. 